\title{
Longitudinal Molecular Imaging of Progesterone Receptor Reveals Early Differential Response to Endocrine Therapy in Breast Cancer with an Activating ESRI Mutation
}

\author{
Manoj Kumar ${ }^{1}$, Kelley Salem¹ ${ }^{1}$ Justin J. Jeffery ${ }^{2}$, Yongjun Yan $^{1}$, Aparna M. Mahajan², and Amy M. Fowler ${ }^{1,2,4}$ \\ ${ }^{1}$ Department of Radiology, University of Wisconsin School of Medicine and Public Health, Madison, Wisconsin; ${ }^{2}$ University of \\ Wisconsin Carbone Cancer Center, Madison, Wisconsin; ${ }^{3}$ Department of Pathology and Laboratory Medicine, University of \\ Wisconsin School of Medicine and Public Health, Madison, Wisconsin; and ${ }^{4}$ Department of Medical Physics, University of Wisconsin \\ School of Medicine and Public Health, Madison, Wisconsin
}

\begin{abstract}
Activating mutations in the estrogen receptor (ER) a-gene (ESR1) result in constitutive transcriptional activity in the absence of estrogen and are associated with endocrine resistance in metastatic ER-positive $(+)$ breast cancer. It is not known how activating ESR1 mutations may alter the predictive values of molecular imaging agents for endocrine therapy response. This study investigated the effect of an activating ESR1 mutation on pretreatment ${ }^{18} \mathrm{~F}$-fluoroestradiol $\left({ }^{18} \mathrm{~F}\right.$ FES) uptake and early assessment of endocrine therapy response using ${ }^{18} \mathrm{~F}-\mathrm{FDG}$ and ${ }^{18} \mathrm{~F}$-fluorofuranylnorprogesterone ( $\left.{ }^{18} \mathrm{~F}-\mathrm{FFNP}\right)$ $\mathrm{PET} / \mathrm{CT}$ imaging of tumor glucose metabolism and progesterone receptor (PR) expression, respectively. Methods: ER+, PR+ T47D breast cancer cells expressing wild-type (WT) ER or an activating ESR1 mutation, Y537S-ER, were used to generate tumor xenografts in ovariectomized female immunodeficient mice supplemented with $17 \beta$-estradiol. Tumor growth curves were determined in the presence or absence of estrogen and for ethanol vehicle control or fulvestrant treatment, a selective ER degrader. Pretreatment ${ }^{18} \mathrm{~F}$-FES uptake was compared between Y537S-ER and WT-ER tumors. Longitudinal $\mathrm{PET} / \mathrm{CT}$ imaging with ${ }^{18} \mathrm{~F}-\mathrm{FFNP}$ and ${ }^{18} \mathrm{~F}-\mathrm{FDG}$ was performed before and 7-9 d after the start of endocrine therapy with fulvestrant. Radiopharmaceutical uptake in Y537S-ER and WT-ER tumors was compared between baseline and follow-up scans. Statistical significance was determined using paired $t$ testing for longitudinal imaging and 2way ANOVA for the ${ }^{18} \mathrm{~F}-\mathrm{FFNP}$ tissue biodistribution assay. Results: Y537S-ER xenografts showed estrogen-independent growth, whereas WT-ER tumors grew only with estrogen. Fulvestrant treatment for 28 d significantly reduced tumor volumes for WT-ER but only stabilized volumes for Y537S-ER. Baseline ${ }^{18} \mathrm{~F}-\mathrm{FES}$ uptake did not significantly differ between WT-ER and Y537S-ER tumors. Fulvestrant treatment induced a similar early metabolic response for both WTER and Y537S-ER tumors. ${ }^{18}$ F-FFNP uptake in WT-ER tumors was significantly reduced after $7 \mathrm{~d}$ of fulvestrant treatment; however, this reduction did not occur in Y537S-ER tumors, which showed no significant change between baseline and follow-up PET/CT. Conclusion: Molecular imaging of PR expression dynamics could be a noninvasive approach for early identification of reduced effectiveness of endocrine therapy resulting from activating ESR1 mutations.
\end{abstract}

Received May 16, 2020; revision accepted Jul. 20, 2020.

For correspondence or reprints contact: Amy M. Fowler, University of Wisconsin School of Medicine and Public Health, 600 Highland Ave., Madison, WI 53792.

E-mail: afowler@uwhealth.org

Published online Aug. 28, 2020.

COPYRIGHT (C 2021 by the Society of Nuclear Medicine and Molecular Imaging.
Key Words: ESR1 mutation; fulvestrant; ${ }^{18} \mathrm{~F}-\mathrm{FFNP}$ PET/CT; ${ }^{18} \mathrm{~F}-$ FDG; ${ }^{18} \mathrm{~F}-\mathrm{FES}$

J Nucl Med 2021; 62:500-506

DOI: $10.2967 /$ jnumed.120.249508

$\mathbf{E}$

strogen receptor (ER) and progesterone receptor (PR) are expressed in the majority of breast cancer cases and are prognostic and predictive biomarkers. Immunohistochemical assessment of these biomarkers is performed clinically to determine whether endocrine therapy should be used. Patients with metastatic ER-positive (+) or $\mathrm{PR}+$ breast cancer can be treated with various endocrine therapy agents such as aromatase inhibitors or selective ER modulators such as tamoxifen. Selective ER degraders are another option; these are pure ER antagonists, competitively inhibiting ER binding with estrogen and targeting ER protein for proteasome-mediated degradation. Fulvestrant is the only Food and Drug Administration-approved drug in this class for the treatment of ER + metastatic breast cancer. Although most patients with metastatic ER + breast cancer have a favorable response to endocrine therapy initially, the response rate decreases with subsequent lines of therapy, indicating the development of resistance (1).

Up to $40 \%$ of patients with metastatic ER + breast cancer treated with endocrine therapy have acquired ER $\alpha$-gene (ESRl) somatic mutations, which correlate with reduced survival (2,3). Most ESR1 mutations occur at amino acids 537 and 538 in the ligandbinding domain (4). These mutations mimic an agonist-bound receptor conformation, resulting in constitutive transcriptional activity in the absence of estrogen $(5,6)$. Of the reported mutations, tyrosine-537-serine (Y537S) has a high prevalence, has maximal estrogen-independent transcriptional activity, and is more resistant to ER antagonists $(7,8)$. The conformational change in the ligandbinding domain also impacts ligand-binding affinity, with reduced binding of Y537S-ER to estradiol and fulvestrant compared with wild-type (WT) ER $(4,7)$. Higher doses of fulvestrant are required to inhibit transcriptional activity and cell proliferation in Y537S-ER than in WT ER (7-11). Emergence of ESRl tumor mutations driving endocrine resistance is a significant problem because there are currently no clinically approved therapies that target ESR1 mutations.

Clinical studies of imaging biomarkers to predict response to endocrine therapy have focused primarily on ER using ${ }^{18} \mathrm{~F}$-fluoroestradiol $\left({ }^{18} \mathrm{~F}-\mathrm{FES}\right)$ and glucose metabolism using ${ }^{18} \mathrm{~F}-\mathrm{FDG}(12-18)$. 
Preclinical studies have also identified ${ }^{18} \mathrm{~F}$-fluorofuranylnorprogesterone, ${ }^{18} \mathrm{~F}$-FFNP, a radiolabeled progestin analog that binds to PR, as a potential biomarker of estrogen sensitivity and endocrine therapy response (19-21). PR is a downstream target of activated ER and an indicator of ER functionality such that when ER transcriptional function is blocked, PR protein expression decreases. A study investigating ${ }^{18} \mathrm{~F}$-FFNP PET imaging in breast cancer has demonstrated its safety, dosimetry, and correlation between uptake and tumor PR status, thus supporting its translational potential (22).

It is not known how activating ESR1 mutations in ER+ metastatic breast cancer may alter the predictive values of molecular imaging agents for endocrine therapy response. In this study, we investigated how expression of Y537S-ER impacts the predictive values of ${ }^{18} \mathrm{~F}$-FES, ${ }^{18} \mathrm{~F}$-FDG, and ${ }^{18} \mathrm{~F}$-FFNP. Given the constitutive transcriptional activity and reduced endocrine sensitivity of the mutant receptor, we hypothesized that suppression of ${ }^{18} \mathrm{~F}$-FFNP uptake and inhibition of tumor glycolytic activity will be impaired in tumors expressing Y537S-ER treated with fulvestrant.

\section{MATERIALS AND METHODS}

\section{Cell Culture}

The experiments adhered to a protocol approved by the Office of Biologic Safety. CRISPR-Cas9 genome-edited T47D cells with WT and ESR1 knock-in of Y537S were kindly provided by Dr. Steffi Oesterreich (University of Pittsburgh) (10) and tested negatively for murine pathogens and Mycoplasma (IDEXX BioResearch). Cells were cultured in RPMI medium (Corning) with $10 \%$ fetal bovine serum (VWR) and $1 \%$ penicillin/streptomycin (Gibco) at $37^{\circ} \mathrm{C}$ and $5 \% \mathrm{CO}_{2}$. Steroid hormone-depleted conditions consisted of $10 \%$ charcoal/dextran-stripped fetal bovine serum in phenol red-free medium with $1 \%$ penicillin/streptomycin and $2 \%$ L-glutamine.

\section{Droplet Digital Polymerase Chain Reaction (ddPCR)}

Reactions were prepared with $25 \mathrm{ng}$ of extracted DNA (DNeasy Blood and Tissue Kit; Qiagen) in ddPCR supermix for probes (BioRad). The forward ESR1 primer was 5'-GGCATGGAGCATCTGTACAG$3^{\prime}$; the reverse primer was 5'-CAAGTGGCTTTGGTCCGTC-3'. The WT-ESR 1 probe was $5^{\prime}$-HEX/CCCCTCTATGACCTGCTGCT-3'. The Y537S-ESR1 probe was 5'-56-FAM/CCCCTCTCTGACCTGCTGC/ 3IABkFQ-3' (Integrated DNA Technologies). Droplets were generated with a Bio-Rad QX200 Droplet Generator using a $20-\mu \mathrm{L}$ reaction mix with $70 \mu \mathrm{L}$ of droplet generation oil. Droplets were then moved into a 96-well PCR plate and run on a $\mathrm{C} 1000$ Thermal Cycler $\left(95^{\circ} \mathrm{C} \times 10 \mathrm{~min}\right.$, 40 cycles of $94^{\circ} \mathrm{C} \times 30 \mathrm{~s}$ and $60^{\circ} \mathrm{C} \times 1 \mathrm{~min}, 98^{\circ} \mathrm{C} \times 10 \mathrm{~min}$ with a $105^{\circ}$ $\mathrm{C}$ heated lid). PCR products were then subjected to flow cytometry using a QX200 Droplet Reader, measuring the equivalent of 10,000 genomic events. Mutation allele fractions were determined using QuantaSoft Analysis Pro, version 1.0.596 (Bio-Rad).

\section{Mice, Tumor Xenografts, and Treatments}

Animal studies adhered to American Association for Laboratory Animal Science guidelines and followed a protocol approved by the Institutional Animal Care and Use Committee. Seven- to 10-wk-old female immunodeficient athymic nude mice (NCr-nu/nu; Charles River) were used for all experiments except for the ${ }^{18} \mathrm{~F}-\mathrm{FES}$ tissue biodistribution assay, which used NOD scid $\gamma$ (University of Wisconsin-Madison, Biotron Laboratory Breeding Core). Cells $(3,000,000)$ were injected into the second thoracic mammary fat pads at a 1:1 ratio of Matrigel (BD Biosciences) and phosphate-buffered saline for a total volume of $100 \mu \mathrm{L}$. Tumors were measured using calipers, and volume was calculated [(length $\times$ width $\left.^{2}\right) / 2$ ].

To assess estrogen-dependent tumor growth, ovariectomized mice were given a $10 \mu \mathrm{g} / \mathrm{mL}$ concentration of $17 \beta$-estradiol (E2) in the drinking water or regular drinking water (19). For fulvestrant treatment studies, ovariectomized mice were subcutaneously implanted with silastic tubing $(1.98 \mathrm{~mm}$ inner diameter, $3.17 \mathrm{~mm}$ outer diameter, 1.6-cm length; Dow Corning) containing $20 \mu \mathrm{g}$ of E2 (60-d release) to support tumor growth. Mice with palpable tumors ( $>3-\mathrm{mm}$ diameter) were randomized to control and treatment groups. After randomization, we confirmed for each experiment that there was no significant difference in tumor volume between treatment groups. The mice received subcutaneous twice-weekly injections of fulvestrant $(4 \mathrm{mg} /$ mouse; Sandoz) or vehicle control $(100 \mu \mathrm{L}$ of sunflower oil with ethanol).

\section{Radiopharmaceuticals, Tissue Biodistribution, and Imaging}

${ }^{18}$ F-FES and ${ }^{18}$ F-FFNP were synthesized by the University of Wisconsin-Madison Radiopharmaceutical Production Facility (23). ${ }^{18} \mathrm{~F}-\mathrm{FDG}$ was obtained commercially (Sofie). The molar activity of ${ }^{18} \mathrm{~F}$-FFNP ranged from 86 to $475 \mathrm{GBq} / \mu \mathrm{mol}$, and ${ }^{18} \mathrm{~F}$-FES was $200 \mathrm{GBq} / \mu \mathrm{mol}$ at the end of synthesis. ${ }^{18} \mathrm{~F}$-FES and ${ }^{18} \mathrm{~F}$-FFNP tissue biodistribution assays were performed $1 \mathrm{~h}$ after tail vein injection. The injected doses (mean $\pm \mathrm{SD}$ ) of ${ }^{18} \mathrm{~F}-\mathrm{FES}$ and ${ }^{18} \mathrm{~F}-$ FFNP were $1.14 \pm 0.04 \mathrm{MBq}(\sim 30 \mu \mathrm{Ci})$ and $3.17 \pm 0.17 \mathrm{MBq}$ $(\sim 85 \mu \mathrm{Ci})$, respectively. Activity in tissues was measured using a $\gamma$-counter, and data were background-corrected to calculate the percentage injected dose per gram (\% ID/g). Tumor-to-muscle ratio was calculated as the ratio of $\% \mathrm{ID} / \mathrm{g}$ for tumor to averaged $\% \mathrm{ID} / \mathrm{g}$ for left and right quadriceps.

${ }^{18} \mathrm{~F}-\mathrm{FFNP}$ PET/CT was performed at baseline and after $7 \mathrm{~d}$ of fulvestrant treatment. To administer equimolar amounts of ${ }^{18} \mathrm{~F}-\mathrm{FFNP}$, we injected the mice via the tail vein with an average of $3.87 \pm 0.12 \mathrm{MBq}$ $(\sim 104 \mu \mathrm{Ci})$ for baseline imaging and $8.70 \pm 0.35 \mathrm{MBq}(\sim 235 \mu \mathrm{Ci})$ for follow-up imaging. For ${ }^{18} \mathrm{~F}-\mathrm{FDG} \mathrm{PET} / \mathrm{CT}$, fasting mice were injected via the tail vein with $5.77 \pm 0.20 \mathrm{MBq}(\sim 156 \mu \mathrm{Ci})$ at baseline and after $9 \mathrm{~d}$ of fulvestrant. The mice were anesthetized with $2 \%$ isoflurane and scanned supine in the small-animal PET/CT scanner (Inveon; Siemens Preclinical Solutions) $1 \mathrm{~h}$ after injection. The mice that were to undergo ${ }^{18} \mathrm{~F}$-FDG PET/CT remained anesthetized during the 1-h uptake time. Scanning and reconstruction parameters were reported previously (24). Volumes of interest were drawn around the tumors, around the pituitary gland as an internal positive control for estrogen-regulated PR expression (25), and within the quadriceps as nontarget tissue uptake. Quantitative uptake was expressed as maximum $\% \mathrm{ID} / \mathrm{g}$.

\section{Histology}

Excised tumors were fixed in $10 \%$ formalin, embedded in paraffin, and sectioned for staining. The slides were deparaffinized, followed by heat epitope retrieval in citrate buffer $(\mathrm{pH} 6.0)$ for $60 \mathrm{~min}$ at $95^{\circ} \mathrm{C}$. Immunostaining was performed for PR (1:100 NCL-L-PGR-312; Leica Biosystems) and ER (1:100 SP1; Thermo Fisher) using the Vectastain ABC HRP Kit (Vector Laboratories). An experienced breast pathologist masked to treatment group evaluated the percentage of tumor cells with positive staining and the intensity of the staining (none, weak, moderate, or strong).

\section{Statistical Analysis}

For the longitudinal PET/CT studies, paired $t$ tests were used to assess change in radiotracer uptake between the 2 imaging time points for the same tumor within the same mouse. Paired $t$ tests were used to compare ${ }^{18} \mathrm{~F}-\mathrm{FES}$ uptake between tumor types within the same mouse. Two-way ANOVA with Tukey posttesting was used for analysis of the transcriptional activity results and for the ${ }^{18} \mathrm{~F}-\mathrm{FFNP}$ tissue biodistribution assay (GraphPad Prism, version 8). Results are presented as mean \pm SE. A $P$ value of less than 0.05 was considered significant. 


\section{RESULTS}

\section{In Vitro Analysis of CRISPR-Edited T47D Y537S-ER and WT-ER Cells}

The Y537S mutation allele fraction was 50\% in Y537S-ER cells, indicating heterozygous knock-in of the mutation and $0 \%$ in WT-ER cells. A $32.7 \pm 10.2$-fold increase in transcriptional activity was observed in Y537S-ER cells compared with WT-ER in the absence of estrogen $(P=0.0195$; Supplemental Fig. 1; supplemental materials are available at http://jnm.snmjournals.org). Constitutive transcriptional activity observed with Y537S-ER is consistent with published studies $(8,10,24)$.

\section{Effect of Estrogen and Endocrine Therapy on WT-ER and Y537S-ER Tumor Growth}

Although WT-ER tumor xenografts demonstrated sustained growth only with estrogen, Y537S-ER tumors grew with or without estrogen (Fig. 1A). Furthermore, Y537S-ER tumors without estrogen grew faster than WT-ER tumors with estrogen. Consistent with in vitro transcriptional function, the in vivo growth of Y537S-ER tumor xenografts is also estrogen-independent. The Y537S allele fraction was $52 \% \pm 2.5 \%$ and $0.2 \% \pm 0.2 \%$ in Y537S-ER and WT-ER tumors, respectively, indicating that the heterozygous presence of the mutation is maintained when these cells are grown as tumor xenografts.

Given previous work demonstrating reduced binding affinity of Y537S-ER for fulvestrant, with higher doses required to inhibit transcriptional activity and proliferation (7-11), we hypothesized that tumors expressing Y537S-ER would be less sensitive to growth inhibition by fulvestrant than would WT-ER. A significant reduction in WT-ER tumor volume was observed by day 10 for fulvestranttreated mice compared with control mice $(P=0.003)$ (Fig. 1B), with an approximately $46 \%$ total reduction in volume from baseline to the 28-d endpoint. Y537S-ER tumors in the control group continued to increase over time, with an approximately $122 \%$ total increase in volume. Y537S-ER tumor growth was arrested with fulvestrant treatment, but tumor volumes did not decrease. A significant difference in Y537S-ER tumor volumes between treatment groups was observed by day $16(P=0.004)$. Thus, tumor growth responses with fulvestrant treatment differed between WT-ER (reduced tumor volumes) and Y537S-ER xenografts (stable tumor

volumes), indicating that Y537S-ER tumors are less sensitive to growth inhibition by fulvestrant than are WT-ER.

\section{Predictive Value of Baseline ${ }^{18}$ F-FES Uptake for Therapy Response}

Several studies have demonstrated ${ }^{18} \mathrm{~F}$-FES PET imaging as a potential predictive biomarker for endocrine therapy response in patients with metastatic ER + breast cancer $(13,18,26)$. Thus, we examined whether differences in baseline ${ }^{18} \mathrm{~F}$-FES uptake exist that could predict the differential growth response to fulvestrant between tumor types. There was no significant difference in ${ }^{18} \mathrm{~F}$ FES uptake between Y537S-ER and WT-ER tumors (Fig. 2A). The tumor-to-muscle ratio was $4.56 \pm 0.33$ for Y537S-ER tumors and $4.21 \pm 0.44$ for WT-ER tumors $(P=0.2772)$ (Fig. 2B). Uterus uptake was $7.89 \pm 1.01 \% \mathrm{ID} / \mathrm{g}$. Thus, comparable baseline ${ }^{18} \mathrm{~F}-\mathrm{FES}$ uptake in Y537S-ER and WT-ER tumors does not provide insight into their different growth responses to fulvestrant treatment.

\section{Longitudinal ${ }^{18}$ F-FDG PET/CT Assessment of Therapy Response}

Determination of metabolic response by ${ }^{18} \mathrm{~F}-\mathrm{FDG}$ PET/CT has been shown in small clinical studies to be a potential biomarker of endocrine therapy response $(12,15,16,27)$. Thus, we investigated whether differences in metabolic response exist between Y537SER and WT-ER tumors that correspond to the differential growth response to fulvestrant treatment. ${ }^{18} \mathrm{~F}-\mathrm{FDG}$ PET/CT of mice bearing WT-ER and Y537S-ER xenografts was performed before and after $9 \mathrm{~d}$ of fulvestrant treatment, a time point before statistically significant changes in tumor size. Between the baseline and follow-up scans, ${ }^{18}$ F-FDG uptake decreased for both tumor types (Fig. 3; Supplemental Table 1). Reduction in posttreatment ${ }^{18} \mathrm{~F}$ FDG uptake was $-31.85 \% \pm 8.18 \%$ for WT-ER tumors and $-28.29 \% \pm 7.69 \%$ for Y537S-ER tumors $(P=0.7735)$. These data indicate that fulvestrant treatment induces a similar metabolic response in WT-ER and Y537S-ER tumors despite ultimately distinct growth responses.

\section{Longitudinal ${ }^{18} \mathrm{~F}-\mathrm{FFNP}$ PET/CT Assessment of Therapy Response}

We hypothesized that suppression of ${ }^{18}$ F-FFNP uptake would be greater in tumors expressing WT-ER than in Y537S-ER in response to fulvestrant, because of the reduced binding affinity

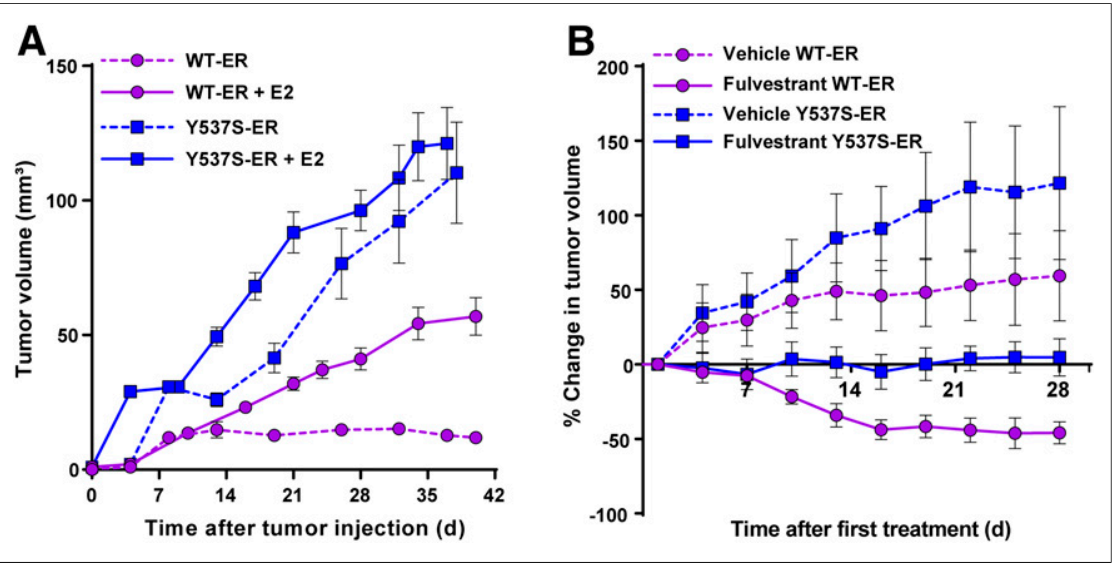

FIGURE 1. Estrogen-independent growth of Y537S-ER tumor xenografts and effect of fulvestrant treatment. (A) WT-ER and Y537S-ER tumor volumes for mice treated with or without $17 \beta-$ estradiol (E2) (10 tumors per group per time point). (B) Percentage change in tumor volumes after treatment with ethanol vehicle control or fulvestrant (12 tumors per group per time point). of Y537S-ER for fulvestrant and reduced treatment efficacy for inhibiting Y537SER transcriptional activity, as previously reported (7-11). ${ }^{18} \mathrm{~F}-\mathrm{FFNP}$ PET/CT of mice bearing WT-ER and Y537S-ER tumor xenografts was performed before and after $7 \mathrm{~d}$ of fulvestrant treatment (Fig. 4; Supplemental Table 2). For WT-ER tumors, ${ }^{18}$ F-FFNP uptake decreased from $3.97 \pm$ $0.35 \% \mathrm{ID} / \mathrm{g}$ at baseline to $2.10 \pm 0.20 \%$ ID/g $(P=0.0001)$ on the follow-up scan. However, there was no significant change in ${ }^{18} \mathrm{~F}-\mathrm{FFNP}$ uptake for Y537S-ER tumors between the baseline $(4.18 \pm 0.37 \% \mathrm{ID} / \mathrm{g})$ and follow-up $(3.92 \pm 0.5 \% \mathrm{ID} / \mathrm{g})$ scans $(P=0.3326)$. Reduction in posttreatment ${ }^{18}$ F-FFNP uptake was $-47.86 \% \pm 2.60 \%$ for WT-ER tumors and $-7.04 \% \pm 8.07 \%$ 


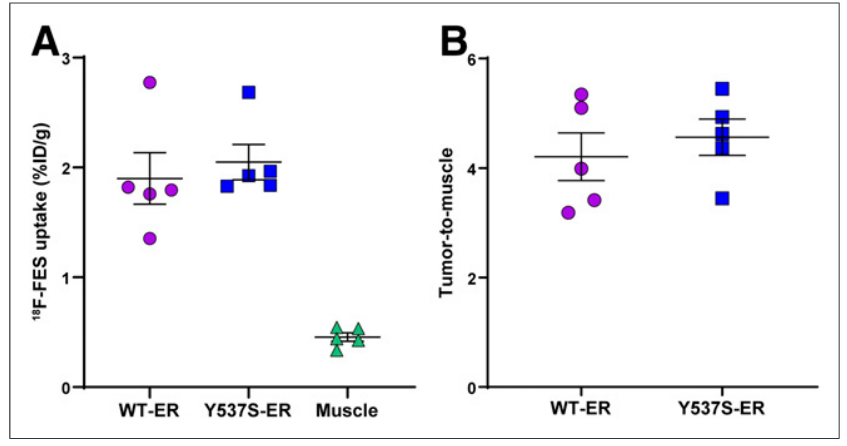

FIGURE 2. Baseline ${ }^{18} \mathrm{~F}-\mathrm{FES}$ uptake in WT-ER and Y537S-ER tumor xenografts: \%ID/g (A) and tumor-to-muscle uptake ratios (B). Estrogen in drinking water was withdrawn $48 \mathrm{~h}$ before ${ }^{18} \mathrm{~F}-\mathrm{FES}$ biodistribution assay.

for Y537S-ER tumors $(P=0.0033)$. As a positive control for fulvestrant inhibition of ER-regulated PR expression, ${ }^{18} \mathrm{~F}-\mathrm{FFNP}$ uptake in the pituitary was reduced to $2.07 \pm 0.04 \% \mathrm{ID} / \mathrm{g}$ during treatment, compared with $3.05 \pm 0.08 \% \mathrm{ID} / \mathrm{g}$ at baseline $(P=$ 0.0003) (Supplemental Fig. 2).

To independently confirm these results, we used a separate cohort of mice bearing Y537S-ER or WT-ER tumor xenografts for a tissue biodistribution assay in which ${ }^{18} \mathrm{~F}$-FFNP uptake was directly measured in excised tumors. For WT-ER tumor-bearing mice treated with fulvestrant for $7 \mathrm{~d},{ }^{18} \mathrm{~F}$-FFNP uptake was less than in the vehicle control group $(1.10 \pm 0.08$ vs. $3.75 \pm 0.35 \%$ $\mathrm{ID} / \mathrm{g}, P=0.0008$ ) (Fig. 5). As with the PET/CT results, there was no significant difference in ${ }^{18} \mathrm{~F}$-FFNP uptake in Y537S-ER tumors between the fulvestrant and control groups $(2.68 \pm 0.13$ vs. 3.24 $\pm 0.35 \% \mathrm{ID} / \mathrm{g}, P=0.8021)$. As a positive control, ${ }^{18} \mathrm{~F}-\mathrm{FFNP}$ uptake in the uterus was lower in the fulvestrant group than in the control group for both Y537S-ER tumor-bearing mice $(P=$ $0.0011)$ and WT-ER tumor-bearing mice $(P<0.0001)$. Excised uteri weighed less in fulvestrant-treated mice than in control mice (WT-ER tumor-bearing mice: $50 \pm 8$ vs. $155 \pm 25 \mathrm{mg}, P=$ 0.0002; Y537S-ER tumor-bearing mice: $60 \pm 4 \mathrm{mg}$ vs. $193 \pm$ $10 \mathrm{mg}, P=0.0001)$, confirming appropriate dosing for ER antagonism.

The PR immunohistochemistry results agreed with ${ }^{18} \mathrm{~F}-\mathrm{FFNP}$ tumor uptake. A decreased percentage of PR-positive cells and reduced staining intensity was observed in WT-ER tumors treated with fulvestrant but not in Y537S-ER tumors (Supplemental Table 3; Supplemental Fig. 3).

\section{DISCUSSION}

The purpose of this study was to determine how altered ER signaling caused by an activating ESRl mutation affects the prediction and early assessment of endocrine therapy response using molecular imaging. We demonstrated distinct growth phenotypes for tumor xenografts expressing Y537S-ER and WT-ER treated with estrogen or fulvestrant endocrine therapy. As expected, WTER tumors were strictly estrogen-dependent for growth, with reduced tumor volumes in response to fulvestrant. In contrast, Y537S-ER tumors did not require estrogen for growth and were less sensitive to growth inhibition by fulvestrant than were WT-ER tumors. Baseline ${ }^{18}$ F-FES uptake did not significantly differ between Y537S-ER and WT-ER tumors. Likewise, early metabolic response was similar between the 2 tumor types, with decreased
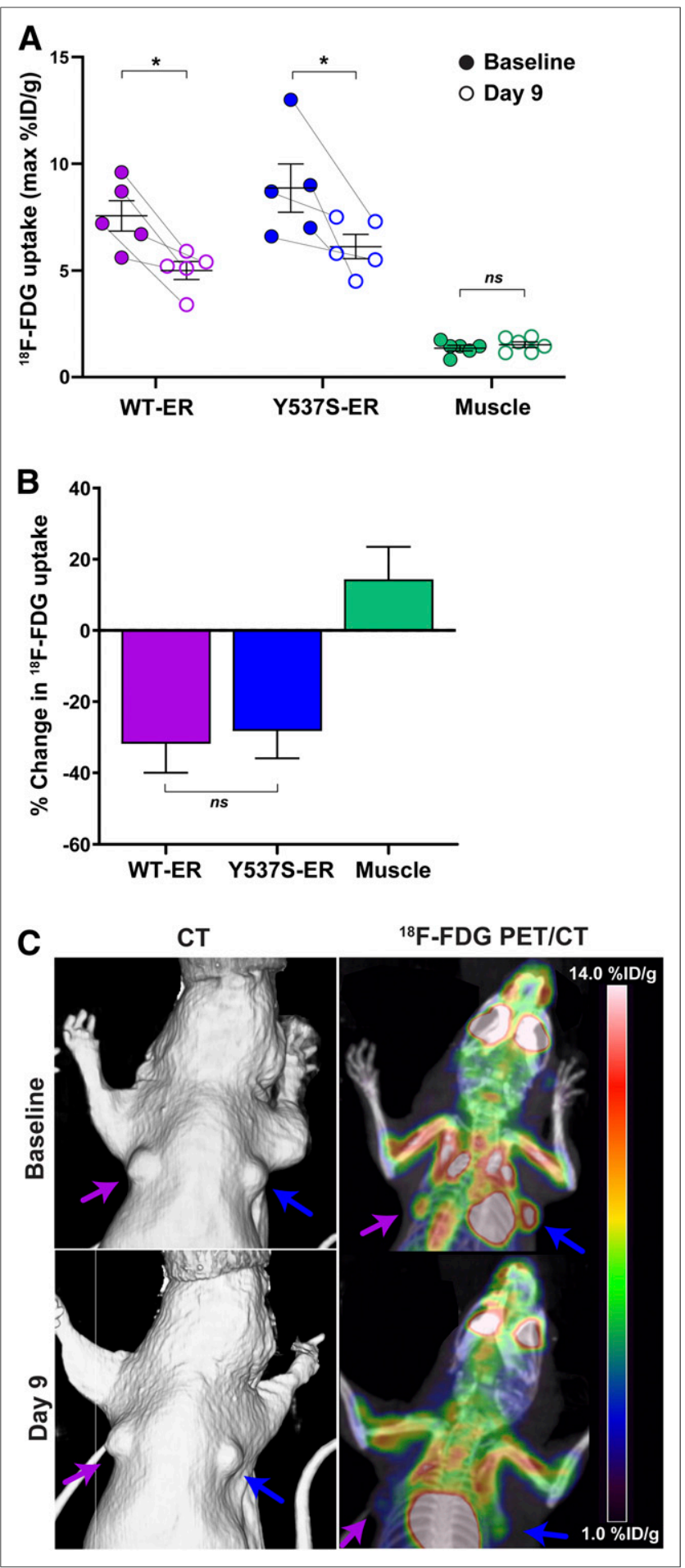

FIGURE 3. ${ }^{18} \mathrm{~F}-\mathrm{FDG}$ PET/CT of WT-ER and Y537S-ER tumor-bearing mice. (A) ${ }^{18} \mathrm{~F}-\mathrm{FDG}$ uptake at baseline and after $9 \mathrm{~d}$ of fulvestrant treatment (5 tumors per group). (B) Percentage change in ${ }^{18} \mathrm{~F}-\mathrm{FDG}$ uptake. (C) Representative PET/CT images. ${ }^{*} P<0.05$. ns $=$ not significant.

${ }^{18} \mathrm{~F}-\mathrm{FDG}$ uptake in response to fulvestrant. However, ${ }^{18} \mathrm{~F}$-FFNP uptake decreased in WT-ER tumors but was persistently elevated in Y537S-ER tumors early after the start of fulvestrant treatment. Differences in ${ }^{18} \mathrm{~F}$-FFNP uptake were observed before changes in tumor size. These results suggest that ${ }^{18} \mathrm{~F}-\mathrm{FFNP}$ PET imaging of PR expression 


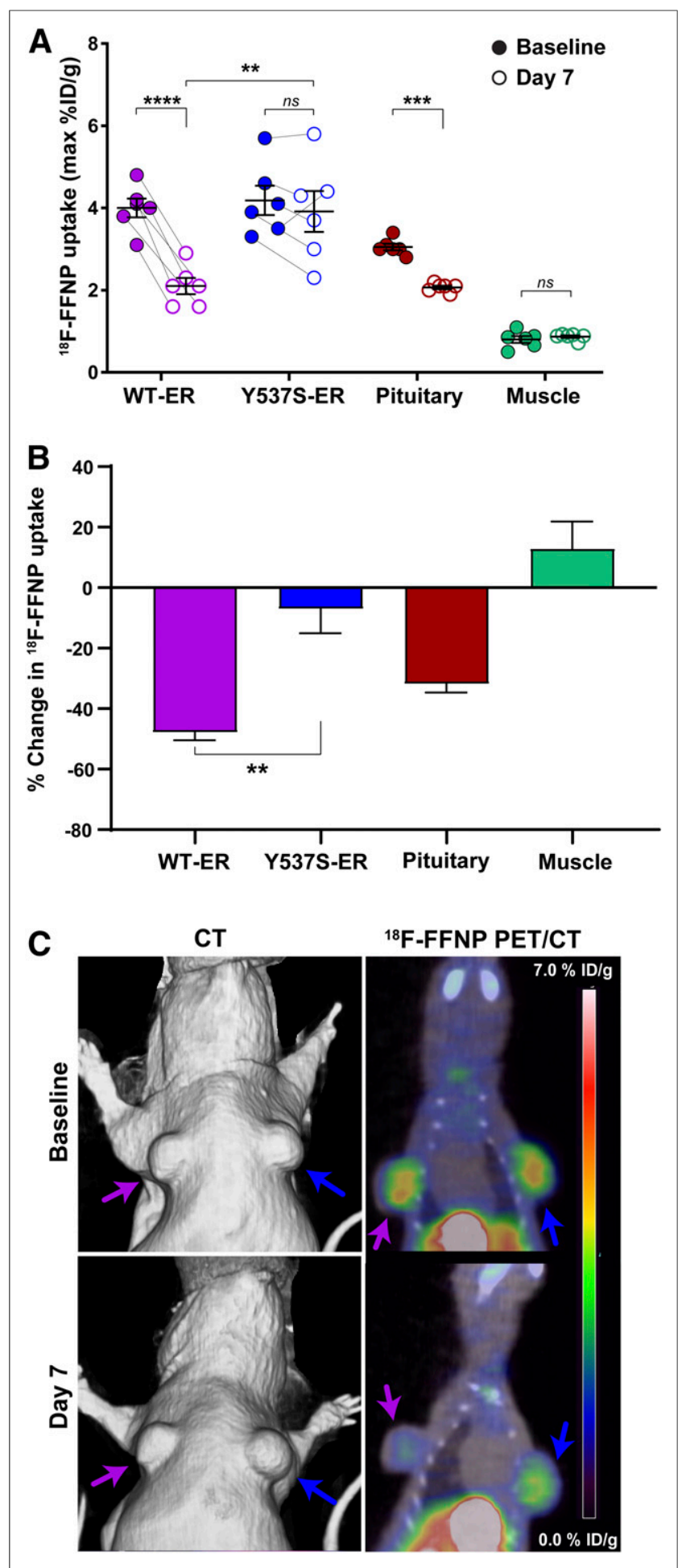

FIGURE 4. ${ }^{18}$ F-FFNP PET/CT of WT-ER and Y537S-ER tumor-bearing mice. (A) ${ }^{18} \mathrm{~F}$-FFNP uptake at baseline and after $7 \mathrm{~d}$ of fulvestrant treatment (6 tumors per group). (B) Percentage change in ${ }^{18} \mathrm{~F}-\mathrm{FFNP}$ uptake. (C) Representative PET/CT images. ${ }^{\star \star} P<0.01$. ${ }^{\star \star \star} P<0.001 .{ }^{* \star \star} P<$ 0.0001. ns $=$ not significant.

dynamics could be an effective approach for early identification of reduced effectiveness of endocrine therapy resulting from activating ESR1 mutations. The noninvasive approach and ability to assess response

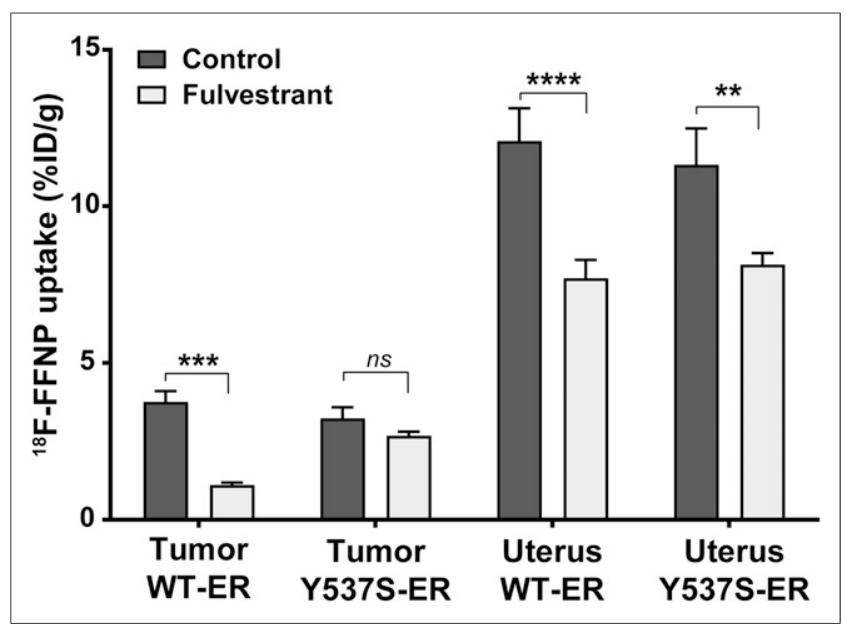

FIGURE 5. ${ }^{18} \mathrm{~F}$-FFNP tissue biodistribution assay for mice bearing bilateral WT-ER tumors (8 tumors; 4 mice per treatment group) or bilateral Y537S-ER tumors (10 tumors; 5 mice per treatment group). ${ }^{\star \star} P<0.01$. ${ }^{\star \star \star} P<0.001$. ${ }^{\star \star \star \star} P<0.0001$. ns $=$ not significant.

across multiple metastatic lesions are advantages of ${ }^{18} \mathrm{~F}-\mathrm{FFNP}$ PET imaging over the repeated biopsies required for PR immunohistochemistry.

The clinical significance of ESR1 mutations has only recently been recognized, and testing is not yet routinely performed. Thus, the tumor ESR1 mutation status in previous clinical PET imaging studies of ER + breast cancer is not known, with the exception of one report published last year. Boers et al. investigated the relationship between intertumoral ${ }^{18} \mathrm{~F}$-FES heterogeneity and time to progression in metastatic ER + breast cancer patients treated with endocrine therapy combined with cyclin-dependent 4/6 kinase inhibition (14). ESR1 mutations were present in circulating tumor DNA from 13 of 23 patients but were not associated with ${ }^{18} \mathrm{~F}-\mathrm{FES}$ uptake (14).

We have previously shown that measurement of ER ligand binding with ${ }^{18} \mathrm{~F}$-FES is not altered in genetically engineered breast cancer xenografts expressing WT-ER or the constitutively active ESR1 mutations, Y537S and Y537C (24). The triple-negative breast cancer model used in our prior study allowed testing of ${ }^{18} \mathrm{~F}-\mathrm{FES}$ binding to mutant receptors in isolation from endogenous WT ER protein (24). ${ }^{18} \mathrm{~F}$-FES results from this study using CRISPR-edited T47D cells with heterozygous ESR1 mutation expression agree with our prior observations. Prospective testing for ESR 1 mutations in future studies of ${ }^{18} \mathrm{~F}$-FES PET imaging is important to validate these findings, particularly with the recent Food and Drug Administration approval of ${ }^{18} \mathrm{~F}-\mathrm{FES}$.

Similar early metabolic responses (reduced ${ }^{18}$ F-FDG uptake) were observed in both WT-ER and Y537S-ER tumors in response to fulvestrant. This observation was unexpected, in view of our hypothesis that Y537S-ER tumors treated with fulvestrant will have less inhibition of glycolytic activity due to reduced endocrine sensitivity of the mutant receptor. Previous preclinical studies have demonstrated that fulvestrant treatment decreases ${ }^{18} \mathrm{~F}-\mathrm{FDG}$ uptake in ER + breast cancer xenografts without known ESR1 mutations. He et al. demonstrated reduced ${ }^{18} \mathrm{~F}$-FDG uptake in ER+ ZR-75-1 tumors after $21 \mathrm{~d}$ of fulvestrant treatment when tumor volumes are decreased compared with vehicle control (28). We also demonstrated that ${ }^{18} \mathrm{~F}$-FDG uptake decreased after 7 and 14 $\mathrm{d}$ of fulvestrant treatment in endocrine-sensitive $\mathrm{ER}+, \mathrm{PR}+, \mathrm{STAT} 1$ (signal transducer and activator of transcription 1)-deficient mouse 
mammary tumors, SSM3, but remained unchanged in endocrineresistant $\mathrm{ER}+, \mathrm{PR}+\mathrm{SSM} 2$ tumors (20). SSM2 tumors were completely resistant to fulvestrant, with the tumor growth rate being identical to that in ethanol vehicle control tumors - an observation that differs from the growth stabilization phenotype of Y537S-ER tumors observed in this study. Thus, the mechanism and magnitude of endocrine therapy resistance appear to affect the metabolic response pattern observed.

These results suggest that early assessment of PR expression dynamics could indicate insufficient inhibition of ER signaling in tumors with activating ESR1 mutations. Persistent ${ }^{18}$ F-FFNP uptake by Y537S-ER tumors after $7 \mathrm{~d}$ of fulvestrant was distinct from the reduced uptake seen with WT-ER tumors, indicating an inadequate therapy response despite maximal ER antagonist dosing. These results agree with our previous preclinical studies demonstrating how changes in ${ }^{18} \mathrm{~F}-\mathrm{FFNP}$ uptake reflect a response to endocrine therapy in ER+, PR+ STAT1-deficient tumors and provide predictive information beyond that provided by ${ }^{18} \mathrm{~F}-\mathrm{FES}$ or ${ }^{18}$ F-FDG PET imaging $(19,20)$. Collectively, ${ }^{18}$ F-FFNP appears to be a robust functional imaging biomarker of endocrine sensitivity and may be generalizable to endocrine therapy resistance acquired through different cellular mechanisms. Clinical trials confirming the predictive value of ${ }^{18} \mathrm{~F}$-FFNP PET imaging in patients, such as a recently completed trial at Washington University (NCT02455453), are important for further validation.

The observed Y537S ESR1 allele fraction in clinical samples from patients with metastatic breast cancer typically ranges between $23 \%$ and $62 \%$, but can be as low as $4 \%$ (8). Thus, the $52 \%$ Y $537 \mathrm{~S}$ ESR1 allele fraction present in the tumor model system used in our study appropriately represents the allele fractions found in metastases. However, the utility of serial ${ }^{18} \mathrm{~F}-\mathrm{FFNP}$ imaging as a biomarker for response to fulvestrant in tumors with low fractions of Y537S ESR1 mutation alleles would need to be directly tested.

This investigation focused on one ESR1 mutation and one endocrine therapy agent. Other activating ESRI mutations may yield distinct results, since mutation site-specific gene regulation and antiestrogen sensitivity have been recognized $(7,10,29)$. Also, this work assessed ${ }^{18}$ F-FES uptake before the start of endocrine therapy, similar to an ongoing multicenter clinical trial (NCT02398773). It is possible that differences in residual ER binding capacity during fulvestrant treatment could be observed between tumors expressing activating ESR1 mutations and WT. Imaging of other pathways, such as glutamine metabolism, could also be informative for assessing therapy response, because Y53S-ER cells were recently shown to have glycolytic rates similar to those of WT-ER cells, but with enhanced mitochondrial activity and glutamine use (30).

\section{CONCLUSION}

These findings suggest that ${ }^{18} \mathrm{~F}-\mathrm{FFNP}$ PET imaging is capable of differentiating endocrine therapy effects in breast cancer with activating Y537S ESR1 mutations. Our results also support ESR1 mutation testing in clinical trials involving molecular imaging, since distinct responses can occur with endocrine therapy depending on mutation status.

\section{DISCLOSURE}

The University of Wisconsin-Madison Department of Radiology receives research support from GE Healthcare. Services were provided by the Translational Research Initiatives in Pathology laboratory, which is in part supported by the University of Wisconsin Department of Pathology and Laboratory Medicine and UWCCC (grant P30 CA014520), the Experimental Pathology Laboratory (grant P30 CA014520), and the University of Wisconsin-Madison Biophysics Instrumentation Facility, established with support from the University of Wisconsin-Madison and grants BIR-9512577 (NSF) and S10 RR13790 (NIH). Financial support was received from the Clinical and Translational Science Award program, through the NIH National Center for Advancing Translational Sciences (grant UL1TR002373), a University of Wisconsin Institute of Clinical and Translational Research KL2 Scholar Award (KL2TR000428), an American Cancer Society Institutional Research Grant Pilot Award (IRG-15-213-51), and a Mary Kay Innovative/Translational Cancer Research Grant. No other potential conflict of interest relevant to this article was reported.

\section{ACKNOWLEDGMENTS}

We thank Dr. Steffi Oesterreich (University of Pittsburgh) for kindly providing cells. We thank the University of Wisconsin-Madison Cyclotron Laboratory for ${ }^{18} \mathrm{~F}$ production, the Radiopharmaceutical Production Facility for ${ }^{18}$ F-FFNP and ${ }^{18}$ F-FES synthesis, and the University of Wisconsin Small Animal Imaging \& Radiotherapy Facility (Cancer Center Support Grant NCI P30 CA014520) for services provided.

\section{KEY POINTS}

QUESTION: How do activating ESR1 mutations affect the predictive values of ${ }^{18} \mathrm{~F}-\mathrm{FES},{ }^{18} \mathrm{~F}-\mathrm{FDG}$, and ${ }^{18} \mathrm{~F}-\mathrm{FFNP}$ as endocrine therapy response biomarkers in $\mathrm{ER}+$ breast cancer?

PERTINENT FINDINGS: ${ }^{18} \mathrm{~F}-\mathrm{FFNP}$ uptake decreased in WT-ER tumors but was persistently elevated in Y537S-ER early after the start of fulvestrant treatment.

IMPLICATIONS FOR PATIENT CARE: Early assessment of PR expression dynamics using ${ }^{18} \mathrm{~F}$-FFNP PET imaging could indicate insufficient inhibition of ER signaling and inadequate therapy in tumors with activating ESR1 mutations.

\section{REFERENCES}

1. Osborne CK, Schiff R. Mechanisms of endocrine resistance in breast cancer. Annu Rev Med. 2011;62:233-247.

2. Chandarlapaty S, Chen D, He W, et al. Prevalence of ESR 1 mutations in cell-free DNA and outcomes in metastatic breast cancer: a secondary analysis of the BOLERO-2 clinical trial. JAMA Oncol. 2016;2:1310-1315.

3. Fribbens C, O'Leary B, Kilburn L, et al. Plasma ESR1 mutations and the treatment of estrogen receptor-positive advanced breast cancer. J Clin Oncol. 2016;34: 2961-2968.

4. Katzenellenbogen JA, Mayne CG, Katzenellenbogen BS, Greene GL, Chandarlapaty S. Structural underpinnings of oestrogen receptor mutations in endocrine therapy resistance. Nat Rev Cancer. 2018;18:377-388.

5. Fanning SW, Mayne CG, Dharmarajan V, et al. Estrogen receptor alpha somatic mutations Y537S and D538G confer breast cancer endocrine resistance by stabilizing the activating function-2 binding conformation. eLife. 2016;5:e12792.

6. Nettles KW, Bruning JB, Gil G, et al. NFkappaB selectivity of estrogen receptor ligands revealed by comparative crystallographic analyses. Nat Chem Biol. 2008;4:241-247.

7. Zhao Y, Laws MJ, Guillen VS, et al. Structurally novel antiestrogens elicit differential responses from constitutively active mutant estrogen receptors in breast cancer cells and tumors. Cancer Res. 2017;77:5602-5613.

8. Toy W, Weir H, Razavi P, et al. Activating ESR1 mutations differentially affect the efficacy of ER antagonists. Cancer Discov. 2017;7:277-287. 
9. Harrod A, Fulton J, Nguyen VTM, et al. Genomic modelling of the ESR1 Y537S mutation for evaluating function and new therapeutic approaches for metastatic breast cancer. Oncogene. 2017;36:2286-2296.

10. Bahreini A, Li Z, Wang P, et al. Mutation site and context dependent effects of ESR1 mutation in genome-edited breast cancer cell models. Breast Cancer Res. 2017;19:60.

11. Fanning SW, Jeselsohn R, Dharmarajan V, et al. The SERM/SERD bazedoxifene disrupts ESR1 helix 12 to overcome acquired hormone resistance in breast cancer cells. eLife. 2018;7:e37161.

12. Mortimer JE, Dehdashti F, Siegel BA, Trinkaus K, Katzenellenbogen JA, Welch MJ. Metabolic flare: indicator of hormone responsiveness in advanced breast cancer. J Clin Oncol. 2001;19:2797-2803.

13. Linden HM, Stekhova SA, Link JM, et al. Quantitative fluoroestradiol positron emission tomography imaging predicts response to endocrine treatment in breast cancer. J Clin Oncol. 2006;24:2793-2799.

14. Boers J, Venema CM, de Vries EFJ, et al. Molecular imaging to identify patients with metastatic breast cancer who benefit from endocrine treatment combined with cyclin-dependent kinase inhibition. Eur J Cancer: 2020;126:11-20.

15. Kurland BF, Gadi VK, Specht JM, et al. Feasibility study of FDG PET as an indicator of early response to aromatase inhibitors and trastuzumab in a heterogeneous group of breast cancer patients. EJNMMI Res. 2012;2:34.

16. Mortazavi-Jehanno N, Giraudet AL, Champion L, et al. Assessment of response to endocrine therapy using FDG PET/CT in metastatic breast cancer: a pilot study. Eur J Nucl Med Mol Imaging. 2012;39:450-460.

17. Kurland BF, Peterson LM, Lee JH, et al. Estrogen receptor binding ( ${ }^{18} \mathrm{~F}-\mathrm{FES}$ PET) and glycolytic activity $\left({ }^{18} \mathrm{~F}\right.$-FDG PET) predict progression-free survival on endocrine therapy in patients with ER+ breast cancer. Clin Cancer Res. 2017;23:407-415.

18. van Kruchten M, de Vries EG, Brown M, et al. PET imaging of oestrogen receptors in patients with breast cancer. Lancet Oncol. 2013;14:e465-e475.

19. Chan SR, Fowler AM, Allen JA, et al. Longitudinal noninvasive imaging of progesterone receptor as a predictive biomarker of tumor responsiveness to estrogen deprivation therapy. Clin Cancer Res. 2015;21:1063-1070.

20. Fowler AM, Chan SR, Sharp TL, et al. Small-animal PET of steroid hormone receptors predicts tumor response to endocrine therapy using a preclinical model of breast cancer. J Nucl Med. 2012;53:1119-1126.
21. Salem K, Kumar M, Yan Y, et al. Sensitivity and isoform specificity of ${ }^{18} \mathrm{~F}-$ fluorofuranylnorprogesterone for measuring progesterone receptor protein response to estradiol challenge in breast cancer. J Nucl Med. 2019;60:220-226.

22. Dehdashti F, Laforest R, Gao F, et al. Assessment of progesterone receptors in breast carcinoma by PET with $21{ }^{18}$ F-fluoro- $16 \alpha, 17 \alpha-\left[(R)-\left(11^{\prime}-\alpha\right.\right.$-furylmethylidene)dioxy]-19-norpregn-4-ene-3,20-dione. J Nucl Med. 2012;53:363-370.

23. Salem K, Kumar M, Kloepping KC, Michel CJ, Yan Y, Fowler AM. Determination of binding affinity of molecular imaging agents for steroid hormone receptors in breast cancer. Am J Nucl Med Mol Imaging. 2018;8:119-126.

24. Kumar M, Salem K, Michel C, Jeffery JJ, Yan Y, Fowler AM. ${ }^{18}$ F-fluoroestradiol PET imaging of activating estrogen receptor-alpha mutations in breast cancer. $J$ Nucl Med. 2019;60:1247-1252.

25. Sánchez-Criado JE, Trudgen K, Millan Y, et al. Estrogen receptor (ESR) 2 partially offsets the absence of ESR1 in gonadotropes of pituitary-specific Esr1 knockout female mice. Reproduction. 2012;143:549-558.

26. Mortimer JE, Dehdashti F, Siegel BA, Katzenellenbogen JA, Fracasso P, Welch MJ. Positron emission tomography with 2-[ $\left.{ }^{18} \mathrm{~F}\right]$ fluoro-2-deoxy-D-glucose and $16 \alpha-\left[{ }^{18} \mathrm{~F}\right]$ fluoro-17ß-estradiol in breast cancer: correlation with estrogen receptor status and response to systemic therapy. Clin Cancer Res. 1996;2:933939.

27. Ueda S, Tsuda H, Saeki T, et al. Early metabolic response to neoadjuvant letrozole, measured by FDG PET/CT, is correlated with a decrease in the Ki67 labeling index in patients with hormone receptor-positive primary breast cancer: a pilot study. Breast Cancer. 2011;18:299-308.

28. He S, Wang M, Yang Z, et al. Comparison of ${ }^{18} \mathrm{~F}-\mathrm{FES},{ }^{18} \mathrm{~F}-\mathrm{FDG}$, and ${ }^{18} \mathrm{~F}-\mathrm{FMISO}$ PET imaging probes for early prediction and monitoring of response to endocrine therapy in a mouse xenograft model of ER-positive breast cancer. PLoS One. 2016;11:e0159916.

29. Jeselsohn R, Bergholz JS, Pun M, et al. Allele-specific chromatin recruitment and therapeutic vulnerabilities of ESR1 activating mutations. Cancer Cell. 2018;33:173-186.

30. Zinger L, Merenbakh-Lamin K, Klein A, et al. Ligand-binding domain-activating mutations of ESR1 rewire cellular metabolism of breast cancer cells. Clin Cancer Res. 2019;25:2900-2914. 